\title{
An Essay on Continued Fractions
}

\author{
Leonhard Euler
}

Translated by

Myra F. Wyman* and Bostwick F. Wyman**

-Dreher High School, Columbia, S.C.

*The Ohio State University, Department of Mathematics, 231 West 18th Avenue, Columbus, Ohio 43210

\section{Introduction}

The paper translated here, "De Fractionibus Continuis Dissertatio", represents Euler's first published work on the theory of continued fractions, a subject to which he often returned during his long career. The emphasis is on basic identities and analytic theory, culminating in the development of the continued fraction expansion for $e$. A discussion of the periodic continued fractions associated with quadratic irrationalities is also included, but applications to number theory come in later publications.

Readers interested in mathematical system theory will be concerned primarily with Euler's treatment of the Riccati equation, Sections 28-33. This aspect of the paper served as the chief motivation for the translation project.

We are grateful to $\mathrm{C}$. I. Byrnes for suggesting that we translate this paper, and for his continuing support. Thanks for comments and help with references also to C. Bernlohr, P. Fuhrmann, W. Gragg, A. Lindquist, C. Martin, L. F. Meyers, A. Weil, H. Wimmer, and especially to J. Burckhardt and G. Mislin of the Euler Commission. As far as the translators know, no translation into English or another modern language has been published.

\section{Bibliographical Notes}

Two bibliographies are included. The first is a selected list of sonte of Euler's papers on continued fractions as well as some relevant to the Riccati equation but not necessarily involving tontinued 
Iractions. The second is a bibliography of recent works on continued fractions and sonic background materials on Euler. In particular, $[1,9,14,15]$ fall in this Jatter calcgory. Sce $[2]$ for an overvicw of many of the relevant papers.

References to Euler's published works are given according to the number assigned in Gustav Encstrobm's index:

G. Enestsom, Verzeichnis der Schriften Leonhard Eulers, Jahresbericht der Deutschen Mathematiker-Vereinigung, Ergánzungsband 4, 1. Liefenung 1910, 2. Lieferung 1913.

Papers are cited in the Collected Works compiled by the Swiss Academy of Sciences:

Leonhardi Euleri Opera Omnia: Series I-IV

For example, the present paper is cited E71, O.O. I.14, 1744. That is, Enestrom number 71, Opera Omiria. Series I, Volume 14, originally published in 1744 . Dates are particularly tricky, since the date of composition is often much earlier than the date of publication. This Essuy was written in 1737, but only puhlished seven years later in the Proceedings of the National Acodemy of St. Peterstherg.

1. Various quantities have been considered in Analysis which are easy to express but difficult to evaluate. Namely, irrational and transcendental quantities, among which are logarithms, circular arcs, and the lengths of other curves, are frequently expressed by infinite series. Since the terms of these series are known, they indicate the values of these quantities distinctly enough. Moreover these series are of two kinds, to the first of which belong those whose terms are related by addition or subtraction and to the second those whose terms are related by multiplication. Thus, the area of a circle with diameter 1 may be expressed by either method; according to the first, the area of the circle is

$$
1-\frac{1}{3}+\frac{1}{5}-\frac{1}{7}+\frac{1}{9}-\text { etc. to infinity. }
$$

According to the second method, the area equals the expression

$$
\frac{2 \cdot 4 \cdot 4 \cdot 6 \cdot 6 \cdot 8 \cdot 8 \cdot 10 \cdot 10}{3 \cdot 3 \cdot 5 \cdot 5 \cdot 7 \cdot 7 \cdot 9 \cdot 9 \cdot 11} \text { etc. 10 infinity. }
$$

Of these series, those are to be preferred which converge most quickly and require the fewest terms to supply a good approximation to the desired quantity.

2. In addition to the two kinds of series presented above, we consider a third whose terms are connected by continuing division, so that it will be convenient to call these series continued fractions. Although this construction is less used than the other two, not only does it exhibit its value just as clearly but it is also very well suited to approximate computation. However, these continued fractions have been used so little that except for a few special cases no methods have been available either for computing their precise values or for using them to express given transcendental quantities. Since I have been studying continued fractions for a long time, and I have observed many important facts pertaining both to their use and their derivation, I have decided to discuss them here. Although I have not yet arrived at a complete theory, I believe that these partial 
results which I found after hard work will surely contribute to further study of this subject.

3. In order that what I mean by the name continued fraction will be more clearly understood, I exhibit first of all the most general example:

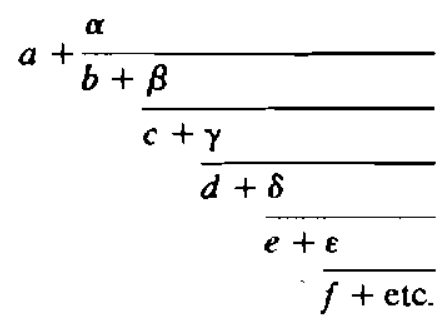

The significance of this expression is easily understood from this method of writing. Clearly this quantity consists of two members, a whole number $a$ and a fraction whose numerator is $\alpha$ and whose denominator is again composed of two members- $a$ whole number $b$ and a fraction whose numerator is $\beta$ and whose denominator again consists of two members, namely $c$ and a fraction as before, and so on to infinity. Here pairs of quantities occur which I have distinguished by letters taken from the Latin and Greek alphabets. Of these quantities those which I have denoted with Greek letters I will call numerators because in fact they constitute the numerators of the sequence of fractions considered above. The remaining quantities expressed in Latin letters will be called denominators, since all of them except the first are indeed part of the denominators of the sequence of fractions.

4. As far as I know, Viscount Brouncker (a) was the first who presented a continued fraction in this way. After Wallis communicated a result on the area of a circle to him, Brouncker changed the expression involved and asserted that the area of a circle is to the square of its diameter as 1 is to

$$
1+\frac{1}{2+\frac{9}{2+\frac{25}{2+\frac{49}{2+\frac{81}{2+\text { etc }}}}}}
$$

where the numerators are squares of odd integers and the denominators are all equal to 2. However, the way in which Brouncker arrived at this expression is not written down, and it would indeed be a pity if this method should perish since doubtless many outstanding facts of this sort can be derived in the same way. Moreover, when Wallis examined this fraction, he tried to produce a proof which is less natural and seems to be wholly different from my method. Moreover, 
Wallis derived the whole approach from the following theorem, namely (b)

$$
\begin{aligned}
a^{2}=(a-1)+\frac{1}{2(a-1)+\frac{9}{2(a-1)+\frac{25}{2(a-1)+\text { etc. }}}} \\
\times(a+1)+\frac{1}{2(a+1)+\frac{9}{2(a+1)+\frac{25}{2(a+1)+\text { etc. }}}}
\end{aligned}
$$

whose truth he confirmed by induction, but most importantly, he did not offer the analysis which led to the theorem.

5. Not only is it possible to approximate the value of a given continued fraction of this kind easily and conveniently, but it is also possible to find bounds between which the true value is contained. Thus, if any quadratic or other transcendental quantity were expressed in this manner, it will be an easy matter to compute it approximately. I shall show this from the form of a general continued fraction:

$$
a+\frac{\alpha}{b+\frac{\beta}{c+\frac{\gamma}{d+\frac{\delta}{e+\text { etc. }}}}}
$$

in which 1 assume all the terms positive. Moreover, it appears that an approximate value is obtained if the continued fraction is broken off at some point, and that the longer the fraction is continued before truncation, the better the approximation obtained.

Thus, $a$ alone (neglecting the entire fractional part) is less than the value of the continued fraction. On the other hand,

$$
a+\frac{\alpha}{b}
$$

has a value larger than the true value, since $b$ is smaller than the complete denominator. But if

$$
a+\frac{\alpha}{b+\frac{\beta}{c}}
$$


is considered, its value is smaller than the true value since the fraction $\beta / c$ makes the denominator $b+\beta / c$ too large. And so in this manner successive truncations of the continued fraction lead to alternately greater and lesser values. Therefore it will be possible to approach the true value of the continued fraction as closely as desired.

6. The following sequence of expressions will be therefore obtained:

$$
a \quad a+\frac{\alpha}{b} \quad a+\frac{\alpha}{b+\frac{\beta}{c}} \quad a+\frac{\alpha}{b+\frac{\beta}{c+\frac{\gamma}{d}}}
$$

of which those of odd order (first, third, fifth,...) are less than the value of the continued fraction, while those of even order are greater than that value. Since the third term is greater than the first, the fifth is greater than the third, and so on, the odd terms by increasing eventually attain the true value of the continued fraction. Similarly the even terms which continually decrease, will by decreasing at length descend to the true value. Moreover, if these expressions are changed in to simple fractions, the following sequence of the same expressions will appear:

$$
\frac{a}{1} \quad \frac{a b+\alpha}{b} \quad \frac{a b c+\alpha c+\beta a}{b c+\beta} \quad \frac{a b c d+a c d+\beta a d+\gamma a b+\alpha \gamma}{b c d+\beta d+\gamma b} \quad \text { etc. }
$$

If this sequence is inspected rather attentively, the law by which these terms progress will easily be understood. By this operation, these fractions can be continued as far as desired without laborious reduction of those composite fractions.

7. The law of progression of these fractions is seen clearly from the following scheme:

$$
\begin{array}{ccccc}
a & b & c & d & c \\
\frac{1}{0} & \frac{a}{1}, & \frac{a b+\alpha}{b}, & \frac{a b c+\alpha c+\beta a}{b c+\beta}, & \frac{a b c d+\alpha c d+\beta a d+\gamma a b+\alpha \gamma}{b c d+\beta d+\gamma b}, \\
\alpha & \beta & \gamma & \delta & \varepsilon
\end{array}
$$

Indeed in these fractions, the denominators of the continued fraction are written above and the numerators are written below. The fraction $1 / 0$ is placed first, according to the rule to be described below. Now the law of progression consists in this: the product of a numerator with the corresponding upper indicator, added to the product of the preceding numerator with the lower indicator, yields the succeeding numerator. A similar rule applies to the denominators. This law is easily observed from the inspection of these fractions if they are continued further, but it is possible to deduce their nature from the continued fractions themselves; however, I believe there is no point in including the proof here. 
8. If the differences of these fractions are arrived at by subtracting each from the preceding, the following sequence appears:

$$
\frac{1}{0}, \quad-\frac{\alpha}{1 \cdot b}, \quad+\frac{\alpha \beta}{b(b c+\beta)}, \quad-\frac{\alpha \beta \gamma}{(b c+\beta)(b c d+\beta d+\gamma b)} \quad \text { etc. }
$$

Here the formation of the numerators is obvious, but each denominator is formed from the two preceding ones. Therefore when the limit which exhibits the true value of the continued fraction is examined from the beginning (after rejecting $1 / 0$ ), the sum of $a$ and all the differences will be the true value of the proposed continued fraction:

$$
\begin{gathered}
a+\frac{\alpha}{1 \cdot b}-\frac{\alpha \beta}{b(b c+\beta)}+\frac{\alpha \beta \gamma}{(b c+\beta)(b c d+\beta d+\gamma b)} \\
-\frac{\alpha \beta \gamma \delta}{(b c d+\beta d+\gamma b)(b c d e+\ldots)}+\text { etc. }
\end{gathered}
$$

We have, therefore, an infinite series of the first kind whose terms are connected by addition and subtraction, which is equal to the value of the proposed continued fraction. This series converges rapidly and is quite suitable for the approximate computation of its value.

If pairs of terms are combined to avoid alternating signs, the same continued fraction will be found to be equal to the following series:

$$
a+\frac{\alpha c}{1(b c+\beta)}+\frac{\alpha \beta \gamma e}{(b c+\beta)(b c d e+\beta d e+\gamma b e+\delta b c+\beta \delta)}+\text { etc. }
$$

The rule for forming the numerators and denominators of this series is easily derived from the one above. Moreover this series converges very quickly and certainly leads to approximations of the true sum.

9. Therefore, the faster this last discovered sequence converges, the faster the continued fraction itself must be said to converge, since the number of terms in the given sequence corresponds to the number of fractions in the continued fraction. Therefore it is clear that a continued fraction converges fastest when its numerators $\alpha, \beta, \gamma, \ldots$ are small and its denominators $a, b, c_{1} \ldots$ are large. Moreover, we can assume that all these numbers, both tumerators and denominators. are integers; for if they were fractions they could be changed into whole numbers by the known reduction of fractions (namely multiplying numerator and denominator by the same number). Therefore if all the numbers $\alpha, \beta, \gamma, \ldots$ as well as $a, b, c, \ldots$ are assumed integral, the continued fraction will converge fastest if all the numerators $\alpha, \beta, \gamma, \ldots$ are equal to 1 . Then indeed the larger the denominators $a, b, c, d, \ldots$ are, the faster the continued fraction will converge. The numerators can not be less than one, since if any numerator is zero, the continued fraction is broken off there and becomes a finite fraction. The same thing happens if any one of the denominators is set equal to infinity, because just as before the continued fraction will he broken off there and will turn into a finite fraction. 
10. If therefore the following continued fraction, all of whose numerators are $\mathbf{I}$, is proposed:

$$
a+\frac{1}{b+\frac{1}{c+\frac{1}{d+\frac{1}{e+\frac{1}{f+\text { etc. }}}}}}
$$

the following sequence of fractions which only depends on $a, b, c, d, \ldots$ approaches its value:

$$
\begin{aligned}
& \begin{array}{lllll}
a & b & c & d & e
\end{array} \\
& \frac{1}{0}, \quad \frac{a}{1}, \quad \frac{a b+1}{b}, \quad \frac{a b c+c+a}{b c+1}, \quad \frac{a b c d+c d+a d+a b+1}{b c d+d+b} \quad \text { etc. }
\end{aligned}
$$

Clearly, the numerator or denominator of each fraction, multiplied by the index and added to the numerator or denominator respectively, give the numerator or denominator of the succeeding fraction. Therefore the value of this continued fraction is equated to the sum of the following series:

$$
\begin{gathered}
a+\frac{1}{1 \cdot b}-\frac{1}{b(b c+1)}+\frac{1}{(b c+1)(b c d+d+b)} \\
-\frac{1}{(b c d+d+b)(b c d e+\ldots)}+\text { etc. }
\end{gathered}
$$

or to the sum of this one, whose denominators are formed from alternating denominators of the series above:

$$
a+\frac{c}{b c+1}+\frac{e}{(b c+1)(b c d e+d e+b e+b c+1)}+\text { etc. }
$$

11. If in such a continued fraction, all of whose numerators are one, the denominators are fractions, it will be convenient to transform it into another fraction, in which both the numerators and denominators are integers. If we are given a continued fraction of this kind,

$$
a+\frac{1}{\frac{b}{B}+\frac{1}{\frac{c}{C}+\frac{1}{\frac{d}{D}+\frac{1}{\frac{e}{E}+\text { etc. }}}}}
$$


it will be changed into the following form:

$$
a+\frac{B}{b+\frac{B C}{c+\frac{C D}{d+\frac{D E}{e+\text { etc. }}}}}
$$

In a similar way, any continued fraction may be changed into another one whose numerators are all equal to 1 , but whose denominators may be fractions. Namely we have

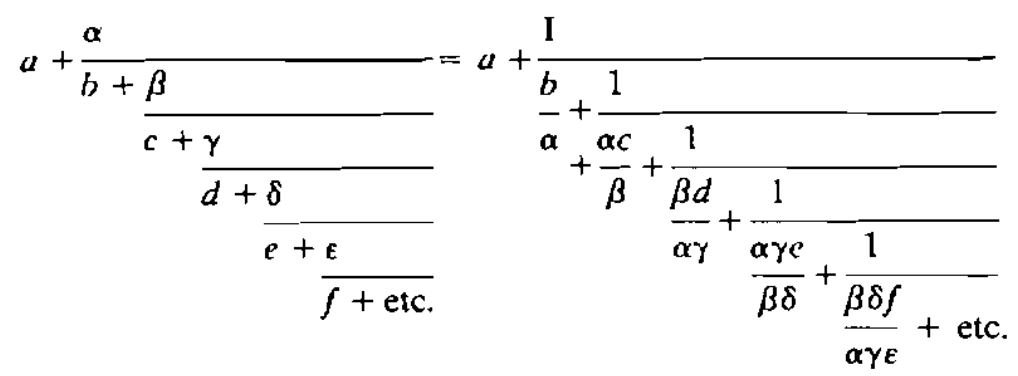

where the second fraction is easily formed from the first.

11a. Therefore, when a continued fraction is given, either its true value (if the continued fraction is truncated), or at least an approximation, can be given as an ordinary fraction. Conversely, an ordinary fraction can be transformed into a continued fraction. The transformation of an ordinary fraction into a continued fraction with numerators all 1 and integral denominators must first be shown. Moreover, every finite fraction whose numerators and denominators are finite whole numbers may be transformed into a continued fraction of this kind which is truncated at a finite level. On the other hand, a fraction whose numerator and denominator are infinitely large numbers (which are given for irrational and transcendental quantities) will go across to a continued fraction running to infinity. To find such a continued fraction, it suffices to assign the denominators, since we set all numerators equal to one. In fact, these will be derived from the numerator and denominator of the given fraction by executing the customary oferation for finding their greatest common divisor. Certainly the numerator is divided by the denominator, and the denominator by the remainder, and thus in turn the preceding divisor by the remainder. In fact, the denominators of the desired continued fraction arise as quotients in this continued division.

12. Thus if it is proposed to change the fraction $A / B$ into a continued fraction all of whose numerators are one, I divide $A$ by $B$ with quotient $a$ and remainder $C$; the preceding divisor $B$ is divided by this remainder $C$ with quotient $b$ and remainder $D$, by which $D$ is divided, and so on until a zero remainder and an infinitely large quotient is obtained. Moreover this operation is 
represented in the following manner:

$B$

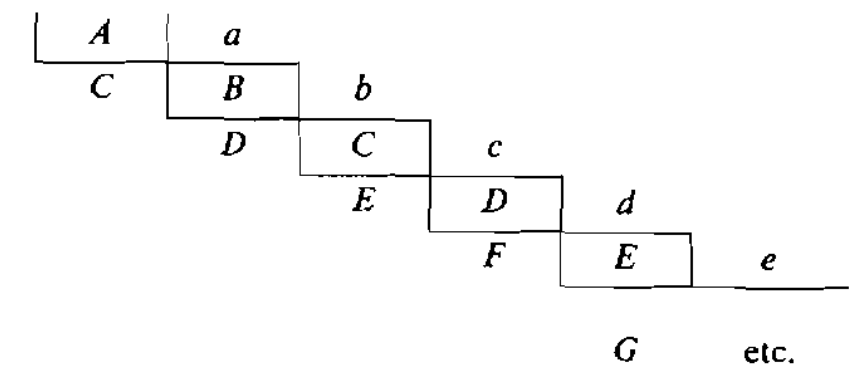

Therefore, the quotients $a, b, c, d, e$, etc., will be found by this operation, and it follows that

$$
\frac{A}{B}=a+\frac{1}{b+\frac{1}{c+\frac{1}{d+\frac{1}{e+e t c}}}}
$$

If the remainder $G=0$, we have

$$
e=\frac{E}{F} \quad \text { and } \quad \frac{1}{e}=\frac{F}{E}
$$

and following this

$$
\begin{aligned}
& d+\frac{1}{e}=d+\frac{F}{E}=\frac{D}{E} \quad \text { and } \quad \frac{1}{d+\frac{1}{e}}=\frac{E}{D} . \\
& c+\frac{1}{d+\frac{1}{e}}=c+\frac{E}{D}=\frac{C}{D} .
\end{aligned}
$$

And ascending by this method up to the beginning, the continued fraction is found to equal $A / B$.

13. If in the fraction $A / B$ we have $A<B$ then the first quotient $a$ is 0 and the first remainder is $A$, so that then $B$ must be divided by $A$. Therefore, in this case

$$
A / \mathrm{B}=\frac{1}{b+\frac{1}{c+\frac{1}{d+\frac{1}{e+e t c}}}}
$$


Moreover, in the case $A<B$ a single term will appear in the continued fraction if $B$ is an integral multiple of $A$, and the continued fraction will consist of two denominators if the ratio $A: B$ belongs to the class of superparticular ratios. On the other hand, there will be more than two denominators if $A: B$ belongs to the class of superpartient ratios (a). Furthermore, the continued fraction will run to infinity if the ratio of $A$ to $B$ is not the ratio of two whole numbers, but is either irrational or transcendental. To change such expressions into continued fractions it is necessary to approximate them by rational numbers, as is customarily done by decimal fractions. Therefore if such expressions are obtained, continued fractions will be formed in the prescribed manner.

14. When some fraction or other expression has been converted into a fraction of this kind, then we can assign an approximate value to it just as in Section 10. Thus, if the expression

$$
\frac{A}{B}=a+\frac{1}{b+\frac{1}{c+\frac{1}{d+\frac{1}{e+\mathrm{etc}}}}}
$$

has heen found and the following sequence of fractions has been constructed from $a, b, c, d$ etc.

$$
\begin{array}{ccccc}
a & b & c & d & e \\
\frac{1}{0}, & \frac{a}{1}, & \frac{a b+1}{b}, & \frac{a b c+c+a}{b c+1}, & \frac{a b c d+c d+a d+a b+1}{b c d+d+b}
\end{array}
$$

then these fractions will be approximately equal to the expression $A / B$, and the further they are from the beginning the closer they are to $A / B$. Moreover, if any of these fractions will be examined, no other fraction which lies closer to the value $A / B$ can be expressed with smaller numbers. In this way the following problem is conveniently solved: "To convert a fraction composed of large numbers into a simpler one which approximates it more closely than can be done with numbers which are no larger." Wallis (a) worked on this problem with great zeal, but he

gave a rather difficult and laborious solution.
15. Our method applies to the given fraction $\frac{355}{113}$

which according to Metius (a) approximately expresses the ratio of circumference to diameter. Therefore we seek fractions made up of the smallest possible numhers which differ only a little from this ratio. Therefore, I divide 355 by 113 and $I$ find

$$
\frac{355}{113}=3+\frac{1}{7+\frac{1}{16}}
$$


from which I form the following fractions

$$
\begin{aligned}
& \begin{array}{lll}
3 & 7 & 16
\end{array} \\
& \frac{1}{0}, \quad \frac{3}{1}, \quad \frac{22}{7}, \quad \frac{355}{113} \text {. }
\end{aligned}
$$

Therefore, the fractions $\frac{3}{1}$ and $\frac{22}{7}$ approximate the fraction $\frac{355}{113}$ more closely than any others made from smaller numbers. Moreover, $\frac{22}{7}$ is larger and $\frac{3}{1}$ is smaller than the given quantity just as we established in general above. These fractions will be called principal convergents, since in addition to these, other non-principal convergents can be assigned which I judge equally satisfactory. Namely, just as the fraction $\frac{22}{7}$ is formed from the preceding ones with the index 7 , so non-principal convergents are formed by substituting smaller numbers in place of 7 which are formed by subtracting the number one over and over.

16. If the ratio of circumference to diameter is computed more exactly by continued division, just as before, the following sequence of quotients appears: 3 , $7,15,1,292,1,1,1,2,1,3,1,14$ etc. from which simple fractions are brought to light in the following way:

$$
\begin{aligned}
& \begin{array}{ccccccc}
3 & 7 & 15 & 1 & 292 & 1 & \\
\frac{1}{0}, & \frac{3}{1}, & \frac{22}{7}, & \frac{333}{106}, & \frac{355}{113}, & \frac{103993}{33102} & \begin{array}{c}
\text { principal } \\
\text { convergents }
\end{array} \\
\hline \frac{2}{1}, & \frac{19}{6}, & \frac{311}{99}, & & \frac{103638}{32989} & \begin{array}{c}
\text { non-principal } \\
\text { convergents }
\end{array}
\end{array} \\
& \frac{1}{1}, \quad \frac{16}{5}, \quad \frac{289}{92}, \quad \frac{103283}{32876} \\
& \frac{13}{4}, \quad \frac{267}{85}, \quad \frac{102928}{32763} \\
& \frac{10}{3}, \frac{245}{78}, \quad \text { etc. } \\
& \frac{7}{2}, \quad \frac{223}{71} \text {, } \\
& \frac{4}{1}, \quad \frac{201}{64} \text {, } \\
& \text { etc. }
\end{aligned}
$$

Therefore, in this way two kinds of fractions are obtained, of which one sort is too large and the other is too small. Namely, those are too large which are written under the indices 3, 15, 292 etc., and the rest arc too small. From this it is easy to establish the whole table of Wallis, which is composed of all fractions approximating the true ratio of circumference to diameter more closely than would be possible with smaller or equal numbers. 
17. By this method it is possible to define the proportion of leap years so that the beginning of ycars will always occur at the same time. This determination depends on the length of the solar year, which according to the most accurate observations I set as 365 days, 5 hours, $49^{\prime}, 8^{\prime \prime}$. Therefore the excess over 365 days will be 5"49'8". and if this excess is set equal to one quarter of a day, each fourth year may safely be declared a leap year. However, since this excess is less than 6 hours, a smaller number of leap years must be accepted. This number is derived from the ratio of $24^{h}$ to $5^{h} 49^{\prime} 8^{\prime \prime}$, or from the fraction $\frac{21600}{5237}$, from which it follows that in the interval of 21,600 years only 5237 leap years ought to be set. Since. however, this period is too large, we shall obtain smaller periods by investigating fractions with smaller numbers which are approximately equal to the fraction $\frac{21600}{5237}$. To this end 1 present the following division:

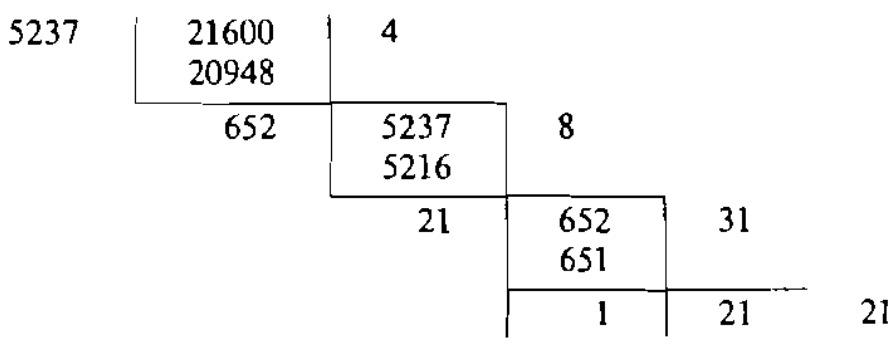

Now from the quotients $4,8,31,21$ whieh are denominators of the continued fraction, the following fractions are formed:

$$
\begin{array}{ccccc}
4 & 8 & 31 & 21 \\
\frac{1}{0}, & \frac{4}{1}, & \frac{33}{8}, & \frac{1027}{249}, & \frac{21600}{5237} .
\end{array}
$$

Of these fractions, the second, $\frac{4}{1}$, gives the ratio of the Julian calendar in which every fourth year is set as a leap year. Therefore the mark may be attained more nearly if in 33 years only 8 years are set as leap years, according to the third fraction. Since, however, it is convenient for the period to have an integral number of years, let us consider non-principal convergents to $\frac{1027}{249}$ which have numerators divisible by 4 . These will be

$$
\frac{136}{33}, \quad \frac{268}{65}, \quad \frac{400}{97}, \quad \frac{532}{129}, \quad \frac{664}{161}, \quad \text { etc. }
$$

of which the third, $\frac{400}{97}$, is most convenient for the computation of the calendar. Moreover, it appears from this that there ought to be only 97 leap years in each interval of 400 years. That is, in this interval, 3 years which were leap years in the 
Julian calendar ought to be changed into ordinary ones-a change which the rules of the Gregorian calendar incorporate. From this, it is understood that a more accurate correction cannot be oblained in a shorter number of years. Most accurately, however, the calendar will be reconciled with the sun if in the interval of 21,600 years one year which ought to be a leap year according to the Gregorian calendar is changed to an ordinary one.

18. We seek now the fractions which approximate $\sqrt{2}$ so closely that no fractions with smaller denominators approach more closely. In fact

$$
\sqrt{2}=1.41421356=\frac{141421356}{100000000}
$$

If continuing division is carried out in the preseribed manner, this fraction gives the quotients

$$
1,2,2,2,2,2,2,2 \quad \text { etc., }
$$

from which the following fractions are formed, satisfactory to such a degree that they are principal or non-principal convergents.

$$
\begin{array}{ccccccccc}
1 & 2 & 2 & 2 & 2 & 2 & 2 & 2 \\
\frac{1}{0}, & \frac{1}{1}, & \frac{3}{2}, & \frac{7}{5}, & \frac{17}{12}, & \frac{41}{29}, & \frac{99}{70}, & \frac{239}{169} & \text { etc. } \\
& & \frac{2}{1}, & \frac{4}{3}, & \frac{10}{7}, & \frac{24}{17}, & \frac{58}{41}, & \frac{140}{99} &
\end{array}
$$

Here the alternate fractions marked by the symbol $\sqrt{ }$ are greater than $\sqrt{2}$, and the remaining ones having the sign 1 are less than $\sqrt{2}$.

19. This description of $\sqrt{2}$ has been suitably presented, since all the quotients except the first have the value 2 , so that

$$
\sqrt{2}=1+\frac{1}{2+\frac{1}{2+\frac{1}{2+\frac{1}{2+\mathrm{etc}}}}}
$$

Similarly, if $\sqrt{3}$ is analyzed, the quotients

$1,1,2,1,2,1,2,1,2,1$, etc. 
are found, so that

$$
\sqrt{3}=1+\frac{1}{1+\frac{1}{2+\frac{1}{1+\frac{1}{2+\frac{1}{1+\frac{1}{2+\text { etc. }}}}}}}
$$

For although it does not follow rigorously from this division that the quotients proceed further in this way, this result not only seems probable but can be proved. We shall show below how to investigate the values of continued Iractions whose denominators are either all equal, or else every other one is equal, or eise every third, etc.

19a. Therefore, let the following continued fraction be given

$$
a+\frac{1}{b+\frac{1}{b+\frac{1}{b+\text { elc. }}}}
$$

which is set equal to $x$. We have

$$
x-a=\frac{1}{b+\frac{1}{b+\frac{1}{b+\text { etc }}}}=\frac{1}{b+x-a}
$$

from which

$$
x^{2}-2 a x+b x+a^{2}-a b=1
$$

and

$$
x=a-\frac{b}{2}+\sqrt{1+\frac{b^{2}}{4}} .
$$


Substituting $b=2$ and $a=1$, this becomes

$$
x=1+\frac{1}{2+\frac{1}{2+\frac{1}{2+\frac{1}{2+\mathrm{etc} .}}}}=\sqrt{2}
$$

if $b=2 a$, we have

$$
\sqrt{a^{2}+1}=a+\frac{1}{2 a+\frac{1}{2 a+\frac{1}{2 a+\frac{1}{2 a+\text { etc. }}}}}
$$

from which it is readily possible to extract an approximate square root of any number which exceeds a square by 1 . Putting $a=2$ supplies the following approximation for $\sqrt{5}$ :

$$
\begin{array}{cccccccc}
2 & 4 & 4 & 4 & 4 & 4 & 4 & \\
\frac{1}{0}, & \frac{2}{1}, & \frac{9}{4}, & \frac{38}{17}, & \frac{161}{72}, & \frac{682}{305}, & \frac{2889}{1292} & \text { etc. } \\
& \frac{1}{1}, & \frac{7}{3}, & \frac{29}{13}, & \frac{123}{55}, & \frac{521}{233}, & \frac{2207}{987} & \\
& & \frac{5}{2}, & \frac{20}{9}, & \frac{85}{38}, & \frac{360}{161}, & \frac{1525}{682} \\
& \frac{3}{1}, & \frac{11}{5}, & \frac{47}{21}, & \frac{199}{89}, & \frac{843}{377}
\end{array}
$$

20. Let the following continued fraction

$$
a+\frac{1}{b+\frac{1}{c+\frac{1}{b+\frac{1}{c+\frac{1}{b+\frac{1}{c+e t c}}}}}}
$$


be set equal to $x$. The value of $x$ itself can be discovered in the following way:

$$
x-a=\frac{1}{b+\frac{1}{c+\frac{1}{b+\frac{1}{c+\frac{1}{b+\frac{1}{\text { etc. }}}}}}}=\frac{1}{b+\frac{1}{c+x-a}} .
$$

Therefore, we have

$$
x-a=\frac{x+c-a}{b x+b c-a b+1}
$$

or,

$$
b x^{2}+b c x-2 a b x=a b c-a^{2} b+c .
$$

Therefore, if $c=2 a$, then

$$
b x^{2}=a^{2} b+2 a \quad \text { and } \quad x=\sqrt{a^{2}+\frac{2 a}{b}}
$$

In a similar way, if

$$
x=a+\frac{1}{b+\frac{1}{c+\frac{1}{d+\frac{1}{b+\frac{1}{c+\frac{1}{d+\text { etc }}}}}}}
$$

then

$$
x-a=\frac{1}{b+\frac{1}{c+\frac{1}{d+x-a}}}
$$


from which it follows that

$$
\begin{aligned}
& (b c+1) x^{2}+(b c d+b+d-c-2 a b c-2 a) x-a b c d+a^{2} b c-a b-a d \\
& \quad+a a-c d+a c-1=0 .
\end{aligned}
$$

In this manner all continued fractions of this kind, whose denominators are either all equal, or else every second or every third or every fourth. etc.. ienoıninator are equal among themselves may be computed. Moreover, every such value $x$ is the root of a quadratic equation.

21. Before we proceed to the computation of continued fractions in general whose denominators form an arithmetic progression let us analyze certain transcendental quantities which, when converted into continued fractions, give denominators proceeding in an arithmetic progression. From these examples a straightforward method of computing continued fractions of this sort will arise. Therefore, testing this method by means of logarithms and other transcendental quantities. $I$ have found that the number whose natural logarithm is 1 , and its powers, lead to continued fractions of this kind. Therefore I set this number $=e$, so that

$$
e=2.71828182845904 \text {, }
$$

which is converted into the continued fraction

$$
e=2+\frac{1}{1+\frac{1}{2+\frac{1}{1+\frac{1}{1+\frac{1}{4+\frac{1}{1+\frac{1}{1+\frac{1}{6+\frac{1}{1+\text { etc }} .}}}}}}}}
$$

whose third denominators make up the arithmetic progression $2,4,6,8$, etc., the others being ones. Even if this rule is seen by observation alone, nevertheless it is reasonable to suppose that it extends to infinity. In fact, this result will be proved below. Likewise, if

$$
\sqrt{c}=1.6487212707
$$


is converted into a continued fraction, it will be

$$
\sqrt{e}=1+\frac{1}{1+\frac{1}{1+\frac{1}{1+\frac{1}{5+\frac{1}{1+\frac{1}{1+\frac{1}{9+\frac{1}{1+\frac{1}{1+\frac{1}{13+\mathrm{etc}}}}}}}}}}}
$$

whose law of progression is similar to the preceding one. Similar rules will be observed in other continued fractions into which powers of $e$ are converted (a).

22. In a similar way, I have considered the cube root of the number $e$, and I have found that

$$
\begin{aligned}
& \frac{\sqrt[3]{c}-1}{2}=0.1978062125=\frac{1}{5+1} \\
& \overline{18+\frac{1}{30+\frac{1}{42+\frac{1}{54+\text { etc. }}}}}
\end{aligned}
$$

All denominators of this continued fraction except the first form an arithmetic progression.

The situation is similar if integral powers of $e$ are considered and transformed into continued fractions. Thus, considering the square of $e, l$ have found (a)

$$
\frac{e^{2}-1}{2}=3.19452804951=3+\frac{1}{5+\frac{1}{7+\frac{1}{9+\frac{1}{11+\frac{1}{13+\frac{1}{15+\text { etc }}}}}}}
$$


Furthermore, from the number $e$ itself, whose continued fraction has an interrupted arithmetic progression of denominators, I have observed that with a few changes of this kind a continued fraction free of interruptions can be formed. For example,

$$
\begin{aligned}
& \frac{e+1}{e-1}=2+\frac{1}{6+1} \\
& 10+1 \\
& 14+1 \\
& \overline{18+\frac{1}{22+\frac{1}{26+\text { etc. }}}}
\end{aligned}
$$

in which there is a regular arithmetic progression proceeding with a difference of 4 .

23. Since 1 have observed a strong similarity between continued fractions whose denominators lie in interrupted or uninterrupted arithmetic progressions, I pose the following question: ls it always possible to transform a continued fraction whose denominators make up an interrupted arithmetic progression into another one whose arithmetic progression is not interrupted? I have considered an arbitrary progression $a, b, c, d$, e, etc., and between each pair in this progression I have inserted the two numbers $m, n$, in order to form the following continued fraction:

$$
a+\frac{1}{m+\frac{1}{n+\frac{1}{b+1}+\frac{1}{n+\frac{1}{c+1}}}}
$$

I have found this fraction to he equal to the following continued fraction, whose denominators lie in an uninterrupted progression:

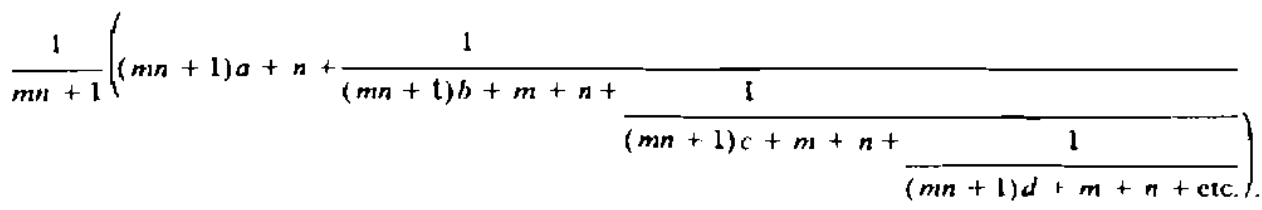


The proof of the equality consists in this: the ordinary fractions which approach the limiting values agree among themselves, so the limits must coincide.

24. If the order of the two interpolated quantities $m, n$ is reversed, the latter continued fraction undergoes a change only in the first term. This yields the following rather elegant theorem:

$$
a+\frac{1}{m+\frac{1}{n+\frac{1}{b+\frac{1}{m+\frac{1}{n+\frac{1}{c+\text { etc. }}}}}}}=\frac{n-m}{m n+1} .
$$

That is, if any numbers are substituted in place of $a, b, c$, $d$, etc., the difference between the two fractions is known and independent of $a, b, c, d, \ldots ;$ namely, it is $\frac{n-m}{m n+1}$.

25. From the same equality found in Section 23 between interrupted and uninterrupted continued fractions, the following equality is found by dividing 1 by each fraction and adding the quantity $A$ to each result.

$$
\begin{aligned}
& A+\frac{1}{a+1} \\
& i n+1 \\
& \overline{n+1} \\
& m+1 \\
& \overline{n+\frac{1}{c+e t c}} \\
& =A+\frac{m n+1}{(m n-1) a+n+} \\
& (m n+1) b+m+n+ \\
& 1 \\
& \overline{(m n+1) c+m+n+\text { etc }} \text {. }
\end{aligned}
$$


Therefore, by the aid of this equation any continued fraction having a progression of denominators interrupted by the two quantities $m$ and $n$ can be converted into another one in which the denominators lie in a progression without any interruptions. Therefore, as we have in the above fractions, $m$ and $n$ are set equal to 1 , the following equation results:

$$
A+\frac{1}{a+\frac{1}{1+\frac{1}{1+\frac{1}{b+\frac{1}{1+\frac{1}{1+\text { etc. }}}}}}}=A+\frac{2}{2 a+1+\frac{1}{2 b+2+\frac{1}{2 c+2+\text { etc. }}}}
$$

From Section 21, we have

$$
\frac{1}{e-2}=1+\frac{1}{2+\frac{1}{1+\frac{1}{1+\frac{1}{4+\text { etc. }}}}}
$$

and setting $A=1, a=2, b=4$, etc., we obtain

$$
\frac{1}{e-2}=1+\frac{2}{5+\frac{1}{10+\frac{1}{14+\frac{1}{18+\frac{1}{22+\text { etc. }}}}}}
$$

It follows by taking reciprocals, that

$$
e=2+\frac{1}{1+\frac{2}{5+\frac{1}{10+\frac{1}{14+\frac{1}{18+\frac{1}{22+\frac{1}{26+\text { etc. }}}}}}}}
$$


In a similar way, we find from the same section that

$$
\begin{aligned}
& \sqrt{e}=1+\frac{1}{1+1} \\
& 1+1 \\
& 1+1 \\
& 5+1 \\
& 1+1 \\
& 1+1 \\
& \overline{9+\text { etc. }} \\
& =1+\frac{2}{3+\frac{1}{12+\frac{1}{20+\frac{1}{28+\text { etc }}}}}
\end{aligned}
$$

These continued fractions converge so fast that it is an easy matter to find the values of $e$ and $\sqrt{e}$ as closely as you please.

26. On the other hand, it is possible to transform a continued fraction whose denominators lie in an uninterrupted progression into another one in which the progression of denominators is interrupted by two constant numbers $m$ and $n$. Thus, 1 have found that

$$
\begin{aligned}
& a+\frac{1}{b+\frac{1}{c+\frac{1}{d+\frac{1}{e+\mathrm{etc}} .}}} \\
& =a-n+\frac{m n+1}{m}+ \\
& \frac{1}{n+\frac{1}{\frac{b-m-n}{m n+1}+\frac{1}{m+\frac{1}{n+\frac{1}{\frac{c-m-n+1}{m n+1}} \frac{1}{m+\text { etc. }}}}}}
\end{aligned}
$$

or, by removing the fractions in the denominators, if the work were examined, it 
will be

$$
\begin{aligned}
& a+\frac{1}{b+\frac{1}{c+\frac{1}{d+\text { etc. }}}} \\
& =a-n+\frac{m n+1}{m+\frac{1}{n+m n+1}} \\
& b-m-n+m n+1 \\
& m+1 \\
& n+m n+1 \\
& c-m-n+m n+1 \\
& m+\frac{1}{n+\text { etc. }}
\end{aligned}
$$

Therefore, setting $m=n=1$,

$$
\begin{aligned}
& a+\frac{1}{b+1} \\
& c+\frac{1}{d+\mathrm{elc}} \text {. } \\
& =a-1+\frac{2}{1+1}
\end{aligned}
$$

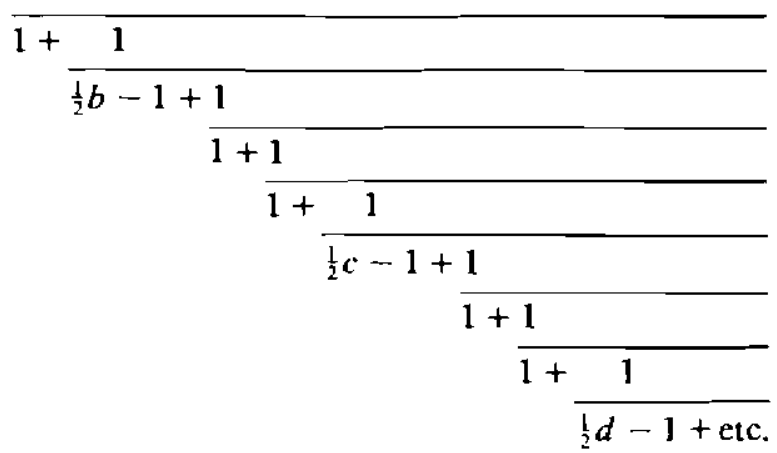

27. We have considered continued fractions whose denominators form an arithmetic progression interrupted in such a way that between any two terms, two constant quantities are interposed. It is possible to extend the same reduction when $4,6,8, \ldots$ constant quantities are interpolated. However, it is not possible to interpolate an odd number of quantities. Thus, if four numbers $m, n, p, q$ are 
interpolated between each contiguous pair of the quantities $a, b, c, d$, setting for short

$$
m n p q+m n+m q+p q+1=P
$$

and

$$
m n p+n p q+m+n+p+q=Q
$$

we obtain

$$
\begin{aligned}
& a+\frac{1}{m+\frac{1}{n+\frac{1}{p+\frac{1}{q+\frac{1}{b+\frac{1}{m+\mathrm{etc} .}}}}}} \\
& =\frac{1}{P}\left(P a+n p q+n+q+\frac{1}{P b}+Q+\frac{1}{P c+Q+\frac{1}{P d+Q+\mathrm{etc} .}}\right)
\end{aligned}
$$

If $m=n=p=q=1$, we obtain

$$
\begin{aligned}
& u+\frac{1}{1+\frac{1}{1+\frac{1}{1+\frac{1}{1+\frac{1}{b+\frac{1}{1+\frac{1}{1+\text { etc. }}}}}}}} \\
& =\frac{1}{5}\left(5 a+3+\frac{\left.1+\frac{1}{5 b+6+\frac{1}{5 c+6}+\frac{1}{5 d+6+e t c .}}\right)}{}\right)
\end{aligned}
$$

From this a new method of converting continued fractions is born.

28. In the preceding sections, where I have converted the number $e$ (whose logarithm is 1) together with its powers into continued fractions, 1 have only 
observed the arithmetic progression of the denominators and I have not been able to affirm anything except the probability of this progression continuing to infinity. Therefore, I have exerted myself in this above all: that I might inquire into the necessity of this progression and prove it rigorously. Even this goal I have pursued in a peculiar way, by which the integration of the equation

$$
a d y+y^{2} d x=x^{\frac{-4 n}{2 n+1}} d x
$$

is reduced to the integration of

$$
a d q+q^{2} d p=d p .
$$

For when I set (a)

$$
p=(2 n+1) x^{\frac{1}{2 n+i}}
$$

I have found that

$$
q=\frac{a}{p}+\frac{1}{\frac{3 a}{p}+\frac{1}{\frac{5 a}{p}+\frac{1}{\frac{7 a}{p}+}}}
$$

Whence, since $q$ can be given in terms of $p$ in case $p=(2 n+1) x^{\frac{1}{2 n+1}}$, it is possible to form a finite equation between $x$ and $y$ which will be the integral of the equation $a d y+y^{2} d x=x^{2 n+1} d x$ for every positive integer $n$.

29. If, therefore, $n$ is assumed to be an infinite number, the expression sought will be a continued fraction running to infinity whose denominators constitute an arithmetic progression. Wherefore the following equation will be obtained

$$
q=\frac{a}{p}+\frac{1}{\frac{3 a}{p}+\frac{1}{\frac{5 a}{p}+\frac{1}{\frac{7 a}{p}+\frac{1}{\frac{9 a}{p}+\text { etc. }}}}}
$$


and $q$ (the value of this continued fraction) will be defined by the equation

$$
a d q+q^{2} d p=d p
$$

In fact, it will be

$$
\frac{a d q}{1-q^{2}}=d p
$$

and

$$
\frac{a}{2} \log \frac{1+q}{1-q}=p+C
$$

where the constant ought to be determined from this equation by setting $q=\infty$ when $p=0$. Wherefore there follows $\frac{a}{2} \log \frac{q+1}{q-1}=p$ and $\frac{q+1}{q-1}=e^{a}$, whence

$$
q=\frac{e^{\frac{2 p}{a}}+1}{e^{\frac{2 p}{a}}-1},
$$

which is the desired value of the continued fraction. Since from this it follows that

$$
e^{\frac{2 p}{a}}=1+\frac{2}{q-1}
$$

we have

$$
e^{\frac{2 p}{a}}=1+\frac{2}{\frac{a-p}{p}+\frac{1}{\frac{3 a}{p}+\frac{1}{\frac{5 a}{p}+\frac{1}{\frac{7 a}{p}+\text { etc. }}}}}
$$

30. If we set $a / 2 p=s$, or $a=2 p s$, there results

$$
\begin{aligned}
& e^{\frac{1}{x}}=1+\frac{2}{2 s-1+} \\
& 6 s+1 \\
& 10 s+1 \\
& \overline{14 s+\frac{1}{18 s+\text { etc. }}}
\end{aligned}
$$


and from the equation found earlier it follows that

$$
\frac{e^{\frac{1}{s}}+1}{e^{\frac{1}{s}}-1}=2 s+\frac{1}{6 s+\frac{1}{10 s+\frac{1}{14 s+\frac{1}{18 s+\text { etc. }}}}}
$$

If the denominators of this fraction are interpolated by two ones, we have

$$
\begin{aligned}
& 1 \\
& \frac{e^{s}+1}{!}=2 s-1+\frac{2}{1+1} \\
& e^{s}-1 \\
& \overline{1+\frac{1}{3 s-1+\frac{1}{1+\frac{1}{1+\frac{1}{5 s-1+\text { etc }}}}}}
\end{aligned}
$$

From which arises the following continued fraction

$$
\begin{aligned}
& e^{\frac{1}{s}}=1+\frac{1}{s-1+1} \\
& 1+1 \\
& 1+1 \\
& 3 s-1+1 \\
& 1+1 \\
& \overline{1+1} \\
& 5 s-1+1 \\
& \overline{1+\text { etc. }}
\end{aligned}
$$

Truly everything found above follows from these formulae, by which we have expressed $e$ and its powers as continued fractions. That is, the necessity of the progressions only observed earlier is now proted.

31. So far we have found a continued fraction whose denominators form an arithmetic progression and whose value can be exhibited. Since however this progression is only a special kind of arithmetic progression I have considered arithmetic progressions in general and continued fractions whose denominators constitute such progressions. I have taken the main idea up again in the following 
way. Suppose therefore given the following continued fraction whose value (which I seek) is set equal to $s$ :

$$
s=a+\frac{1}{(1+n) a+\frac{1}{(1+2 n) a+\frac{1}{(1+3 n) a+\frac{1}{(1+4 n) a+\text { etc. }}}}}
$$

I begin from an approximation to the value of $s$, following the method discussed above:

$$
\begin{array}{cccc}
a & (1+n) a & (1+2 n) a & (1+3 n) a \\
\frac{1}{0}, & \frac{a}{1}, & \frac{(1+n) a^{2}+1}{(1+n) a}, & \frac{(1+n)(1+2 n) a^{3}+(2+2 n) a}{(1+n)(1+2 n) a^{2}+1}, \text { etc. }
\end{array}
$$

These fractions approach the value of $s$, and the fraction carried to infinity will give the true value of $s$.

32. If these fractions are continued further the law by which they are formed will be easily observed. From this law it may he concluded that after hoth the numerator and the denominator are divided by the first term of the denominator, the limiting fraction will be

$$
\frac{a+\frac{1}{1 \cdot n a}+\frac{1}{1 \cdot 2 \cdot 1(1+n) n^{2} a^{3}}+\frac{1}{1 \cdot 2 \cdot 3 \cdot 1(1+n)(1+2 n) n^{3} a^{5}}+\text { ctc. }}{1+\frac{1}{1(1+n) n a^{2}}+\frac{1}{1 \cdot 2(1+n)(1+2 n) n^{2} a^{4}}+\frac{1}{1 \cdot 2 \cdot 3(1+n)(1+2 n)(1+3 n) n^{3} a^{4}}+\text { ctc. }}
$$

to which $s$ is set equal. Therefore I substitute $a=\frac{1}{\sqrt{n} z}$, so that

$$
s=\frac{1}{\sqrt{n} z} \cdot \frac{1+\frac{z}{1 \cdot 1}+\frac{z^{2}}{1 \cdot 2 \cdot 1(1+n)}+\frac{z^{3}}{1 \cdot 2 \cdot 3 \cdot 1(1+n)(1+2 n)}+\text { etc. }}{1+\frac{z}{1(1+n)}+\frac{z^{2}}{1 \cdot 2(1+n)(1+2 n)}+\frac{z^{3}}{1 \cdot 2 \cdot 3(1+n)(1+2 n)(1+3 n)}+\text { etc. }}
$$

from which the value is found. Next, let

$$
t=1+\frac{2}{1 \cdot 1}+\frac{z^{2}}{1 \cdot 2 \cdot 1(1+n)}+\frac{z^{3}}{1 \cdot 2 \cdot 3 \cdot 1(1+n)(1+2 n)}
$$


and

$$
\begin{aligned}
u= & 1+\frac{z}{1(1+n)}+\frac{z^{2}}{1 \cdot 2(1+n)(1+2 n)} \\
& +\frac{z^{3}}{1 \cdot 2 \cdot 3(1+n)(1+2 n)(1+3 n)}+\text { etc. }
\end{aligned}
$$

so that

$$
s=\frac{t}{u \sqrt{n} z} .
$$

From inspection of these two series, it is clear that

$$
d t=u d z
$$

and in a similar way, it may be found that

$$
u d z+n z d u=t d z
$$

From the substitution $t=v u$, with

$$
s=\frac{v}{\sqrt{n} z},
$$

we have

$$
v d u+u d v=u d z,
$$

and

$$
u d z+n z d u=u v d z,
$$

from which it follows that

$$
\frac{d u}{u}=\frac{d z-d v}{v}=\frac{v d z-d z}{n z} .
$$

and the following equation between $z$ and $v$ holds:

$$
n z d v-v d z+v^{2} d z=n z d z,
$$

in which I substitute

$$
v=z^{1 / n} q \text { and } z=r^{n}
$$

so that it will change to

$$
d q+q^{2} d r=n r^{n-2} d r .
$$


From this equation, if $q$ may be determined from $r$ and $r$ is set equal to

$$
r=n^{-1 / n} a^{-2 / n}
$$

then the desired value will be

$$
s=\text { arq } .
$$

33. Therefore, the computation of the value of the given continued fraction, which I have called $s$, with

$$
s=a+\frac{1}{(1+n) a+\frac{1}{(1+2 n) a+\frac{1}{(1+3 n) a+\frac{1}{(1+4 n) a+\text { etc. }}}}}
$$

is reduced to the solution of the equation

$$
d q+q^{2} d r=n r^{n-2} d r .
$$

To find the integral of this equation, note that $a=\infty$ implies $s=\infty$ and $a=0$ implies $s=1$. From this, the following rule for introducing a constant of integration is found, so that in the case $n \leqslant 2$ if $q=\infty$ I set $r=0$. Morcover, we assume that $n$ is a positive number from which a continued fraction arises, having (so far as we have considered) positive denominators.

34. It is established moreover that the derived equation

$$
d q+q^{2} d r=n r^{n-2} d r
$$

agrees with the equation once proposed by Count Riccati and it follows that for $n$ we obtain positive numbers, because only those cases can be integrated in which n is a number of the form $\frac{1}{2 m+1}$, where $m$ is a positive integer. Because of this, the value of the following continued fraction can always be given in a finite expression:

$$
a+\frac{1}{\frac{(2 m+3) a}{2 m+1}+\frac{1}{\frac{(2 m+5) a}{2 m+1}+\frac{1}{\frac{(2 m+7) a}{2 m+1}+\text { etc. }}}}
$$


Indeed, this is obvious. In case $m=0$, we have the continued fraction

$$
a+\frac{1}{3 a+\frac{1}{5 a+\frac{1}{7 a+\frac{1}{9 a+\text { etc. }}}}}
$$

whose value we have already found above. It is possible to ceduce the general case to this one; for if I set $a=(2 m+1) b$ I obtain

$$
(2 m+1) b+\frac{1}{(2 m+3) b+\frac{1}{\frac{1}{(2 m+5) b+\mathrm{etc}} .}}
$$

which is already known as long as $\boldsymbol{m}$ is a positive integer.

35. Therefore, it appears through this resolution of continued fractions that the integration of the equation

$$
d q+q^{2} d r=n r^{n-2} d r
$$

is reduced to the integration of this equation

$$
d q+q^{2} d r=2 d r
$$

in case $n=\frac{2}{2 m+1}$ where $m$ is a positive integer. I have discussed this reduction in Section 28 above in the same way, and from this beginning it is possible to finish the calculation. Moreover, by this reasoning it is possible to figure out how the exact value of this kind of continued fraction is discovered. I shall consider the case $n=2$ (or $m=0$ ). from which

$$
s=a+\frac{1}{3 a+\frac{1}{5 a+\frac{1}{7 a+\mathrm{etc}}}}
$$

The actual value of $s$ is found from the equation

$$
d q+q^{2} d r=2 d r
$$

which when integrated gives

$$
r=\frac{1}{2 \sqrt{2}} \log \frac{q+\sqrt{2}}{q-\sqrt{2}}
$$


from which it follows that

$$
y=\frac{\left(e^{2 r \sqrt{2}}+1\right) \sqrt{2}}{e^{2 r \sqrt{2}}-1} .
$$

\section{Furthermore,}

$$
r=\frac{1}{a \sqrt{2}} \quad \text { and } \quad s=a r q=\frac{q}{\sqrt{2}}
$$

from which the value itself is given as

$$
s=\frac{e^{2 / 4}+1}{e^{2 / a}-1}
$$

just as we found above in Section 29.

\section{Notes}

Notes marked "C. B." are hy Carl Bochm, editor of O.O.f.14. Other notes are by the translaturs Noles are grouped by section.

Note to Title. See also Commentary 123 of this volume, as well as Introduction w the Analysis of the Infinuse, Lausanne 1748, vol. I, chapter 18; O.O.I.8, p. 362. C. B.

The Introductio in Anulisin Infinitorum is also available in a photostat edition published by Culture et Civilisation. Brussels 1967.

4. (a) W. Brouncker (1620)-1684). See J. Wallis, Arithmetted Infinitorum, Oxford 1655, p. 182; Opera Mathematicu, vol. I, Oxford 1695. p. 355 and especially p. 469. See aiso O.O.I.1. p. 507. C.. B.

(b) See E123, O.O.1.14, Scc. 15 C. B.

11. In the first edition the section number 11 was incorrcilly repeated. C. B.

13 (a) Conceming these expressions, compare Martianus Capella (V. Saec). De mipriss Philo

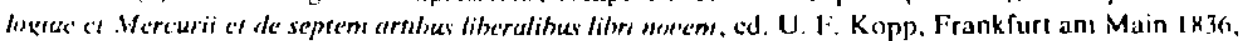

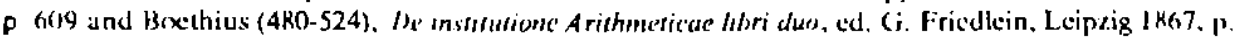
46. C. 13 .

Sce also Boethien Number Theor: A Translatum of the De Insritutione A rishmetica, Michat Mast, Editions Rodopi B. V.. Anustcrdam 1983. U. S. distributor: Humanitics Press, Allantic Highlands, N.J.: pp. 104 and $10 \%$

14. (3) J. Wallis, Opera Marhe'matices, vol. 11, Oxford 1693, thapters 98-99, pp. $418-424$. Sec also chapters S6-81, pp. 232-250. C. B.

15. (a) Adrian Antoniszoon, called by the common name "Metius" (1527-1607). See for example the book of his Adrian Metius (1571-1635) which is called Arithmesicue hbri duo et Geometriae. Hook VI, Lugd. Batav. 1626, lirst part of Geometry, Chapter X. Metius formed the fraction $\frac{355}{113}$ from the fractions $3 \frac{15}{106}$ and $3 \frac{17}{120}$ as follows: $3 \frac{15+17}{106+120}=\frac{355}{113}$. C. B.

17. For more infornation on continued fractions and the calendar, see S. I. Selesthnikov, History of the Calendar and Chrnnology, Izdatel'stvo "Nauka," Moscow, 1970. Chapier 3, swetion I (in Russian). Chapter 3. section 3 on the Roman Calendar contains an explanation of Euler's use of the Latin word "bissexulis" for "Ieap year": extra days in leap years werc insersed after the sixth day. counted up to and including the first of March. We are grateful to L. F. Meycrs for this reference. 
19. In the first edition the number 19 was repcated by nistake. C. B.

21. (a) Ste F. Rudio, Archimedes, Huvgens, Lambert, Iegendre: Vier Abhandlungen üher die Kretsmessung, etc. Leipzig 1892, note 2, p. 51. C. B.

22. (a) A more accurate value is 3.19452804946532 . C. B.

28. (a) Euler had communicated this most ingenious remark to his friend Christian Cioldbach as early as 25 November 1731, Correspondance math. es phl's. publiée par P. H. Fuss. St. Petersburg 1843, vol. I, p. 58, O.O.II1.12. C. B.

A photostatic edition of Fuss 1843 is available from Johnson Reprint Corporation. New York and London, 1968.

34. Jacopo Francesco Riccati, 1676-1754. Bom in Venice, educated at the University of Padua. Ricuati began working on second-order differential equations around 1710 , and conjectured the integrability conditions cited here in 1724 . Prools were supplied by D. Bernoulli and Euler.

\section{Euler Bibliography}

E.11. O.O.I.15, 1733. "On the consiruction of inseparahle differential cquations."

E31, O.0.1.15, 1738. "The construction of the differential equation $a x^{\prime \prime} d x=d y+y^{2} d x$."

E7!, O.O.I.14, 1744. "An essay on continued fractions."

E123, O.O.1.14, 1750. "Ob.cervations on continued Iractions,"

[247, 0.0.I.14, 1760. "On divergent weries [with some remarks on continued fractions.|"

I:260, 0.0.1.15, 1764. "On the progressions of circular ares |rclatted to ctrtain tangents and contenued fractions.".

E2K1. O.O.I.15, 1764. "A model algorithen [for continued fraction computations]."

E2 44, O.O.I.22, 1764. "On the solution of the cquation $d y+a y^{2} d x=b x^{\prime \prime \prime} d x$."

E522, O.O.1.15, 1779. "On the formation of continucd fractions."

E553, O.0.I.15, 1783. "Analytic observations [relating to continued fractions]."

E593, O.O.I.15, 1785. "On the transformation of series into concinued fractions."

Esy4, 0.0.1.18, 1785. "A method for finding integral formulas and summing continucd fractions."

E595, O.0.1.23, 1785. "A method for summing continued fractions which leads to a solution of the Ricuati equation."

I616, O.O.1.16, Part 1, 1788. "On the transformation of a divergent series into a continued fraction."

E742, O.O.1.16, Part 2. 1813. "Observations on special continucd Practions."

E745, O.0.1.16, Part 2.1815. "On the continued fractions of Wallis."

E750, O.O.I.16, Part 2, 1818. "A conmentary on the continued fraction which J acirange used to express binomal powers."

E751, 0.0.1.12, 1818. "An casy method for wolving the Riccali equatioll by means of a continued fraction."

\section{References}

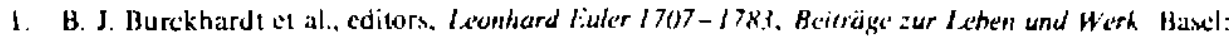
Birkhaüser, 1983.

2. Cieorg Faber, Übersicht üher die Bände 14,15, 16, I6* der crsten Stric, pp. VII-CXII in O.O.1.16, pare 2. Buscl: Teubner. 1935.

3. I' Fuhrmusn. $A$ inatrix Ituclideun algorithn and matrix continued fraction expansions. Si vtums and control tecters. 3 (1983). 263-271.

4. W. H. Gragg and A. Lindquist, On the pirtial realization prohlen. L.mest Algethra ond If. Applecarions, 50. April 19K3. P. 277-319.

$5 \quad$ I:. I.. Ince, Ordmary Differenthal Equatums, Orig. Ed. 1926. Dover Reprint, New York, 1956. (Sec. 7.5, pp. 178 ff.)

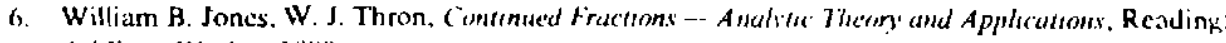
Addison.Wesley. 1980. 
7. R. E. Kalman, On partial realizations, transfer functions, and canonical forms, Acta Potyrechnira Scandinavica, MA 31, Helsinki, 1979, 9-32.

8. O. Perron, Die Lehre un den Kettenbrüsben, (second edition). Leipzig: Teubner, 1919. Reprint, New York: Chclsea, 1950.

9. Mathematics Magazine, A Tribute to Leonhard Euler, 56, No. 5, Nov. 1983.

10. M. A. Shayman, Geometry of the Algebraic Riccati Equation. Parts I and II," SIAM J. Control and Opt., 21. No. 3, May 1983, 375 409.

11. M. E, Van Valkenburg. Introduction to Modern Nerwork Synthesis. New York: Wilcy. 1960). (p. 128; pp. 150-153.)

12. H. S. Wall, Continued Fractions, Now York: Van Nostrand, 1948.

13. (i. N. Watson, A Treatise on the Theory of Bessel Functions, Sccond Edition. New York: MacMillan and Cambridge: University Press, 1944.

14. Andre Weil, Number Theory: An Approrach Through History, Boston: Birkhäuscr, 1984.

15. Harald Wimmer, Leonbard Euler und das Königsberger Brückenproblem--Latein als Sprache der Mathematik im 18. Jabrhundert, Inforntaionen zum Altsprachlichen Unterrichr, 3. Jalirgang. $2 / 1981$.

Received January 30, 1985, and March 8, 1985. 